\title{
Wideband Monopulse Spatial Filtering for Large Receiver Arrays for Reverberant Underwater Communication Channels
}

\author{
Karl F. Nieman, Kenneth A. Perrine, Terry L. Henderson, Keith H. Lent, Terry J. Brudner and Brian L. Evans \\ Advanced Technology Laboratory, Applied Research Laboratories \\ The University of Texas at Austin, Austin, TX 78712 USA
}

\{nieman, perrine, henderson, lent, brudner\}@arlut.utexas.edu and bevans@ece.utexas.edu

\begin{abstract}
Underwater acoustic communication (ACOMMS) is critical for many applications including marine science, oceanographic exploration, offshore surveying/drilling, and military uses. ACOMMS data rates are usually limited by multiple propagation paths with different time delays and Doppler characteristics. It is often difficult to coherently recombine all paths, especially in shallow water, leaving incoherent paths that interfere with the receiver.

One way to suppress unwanted paths is with a directional receiving array. Indeed, many existing large, directional acoustic arrays could be used as ACOMMS receivers. In a number of these arrays, wideband monopulse outputs could be made available. These directional beam outputs, in monopulse pairs, can selectively suppress, or even null, offending multipath when combined with a simple scalar weight. Using an experimental system, we show how a relatively short equalizer, using as inputs the wideband monopulse beam outputs of a large array, can form the backbone of an ACOMMS system that performs effectively in a multipath-limited environment. Our contributions include (i) a multipath-Doppler channel model validated by experimental results, (ii) a receiver design that utilizes monopulse processing, and (iii) an analysis of its performance using simulated and experimental data.
\end{abstract}

\section{INTRODUCTION}

The underwater acoustic channel is bandlimited by absorption loss that arises primarily from the strain relaxation characteristics of the trace solutes magnesium sulfide and boric acid (see Figure 1) [1]. Practical ACOMMS systems typically operate within a generous portion of the absorptionlimited spectrum, due in part to the low ambient background in many underwater communication scenarios [2]. Typical values for short to medium-range ACOMMS systems are shown in Table 1.

The data capacity in many underwater acoustic channels is limited by reverberant scattering rather than ambient noise. Reverberant scattering is caused by the highly anisotropic underwater environment and is commonly grouped into two categories-boundary and volume reverberation. Boundary reverberation is scattering off the surface or bottom, while
TABLE I

TyPiCAL VALUES FOR A SHORT tO MEDIUM RANGe ACOMMS SySTEM

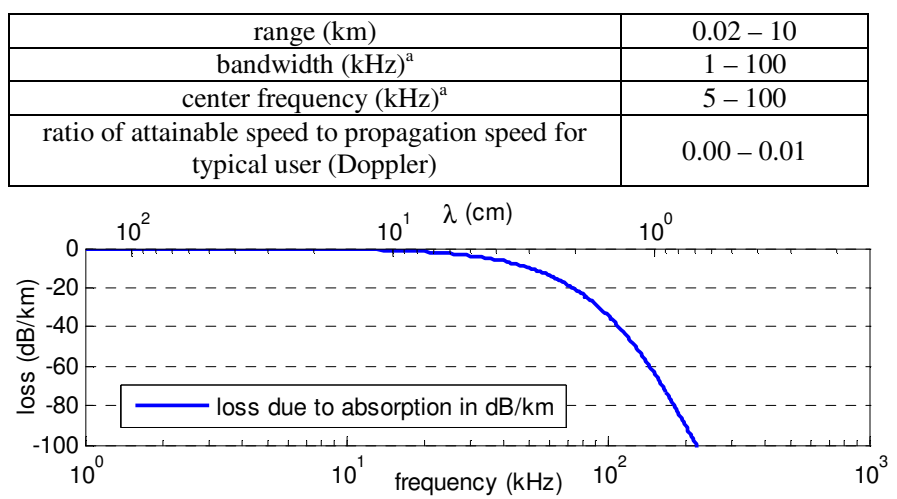

Figure 1. Acoustic loss due to absorption. Calculated using formula in [3].

volume reverberation is scattering within the media, primarily caused by suspended scatterers such as fish. Time-varying paths, strong, non-linear Doppler effects, and long delay spreads further increase channel complexity-e.g. the equivalent propagation delay of an acoustic signal over $2 \mathrm{~km}$ is equivalent to sending an RF signal to the moon $(\sim 384,000$ $\mathrm{km})$. Doppler effects are also very pronounced when compared to other propagation media, since the ratio of attainable speed to acoustic propagation speed is not so small.

For these reasons, many classical digital communication receiver architectures developed for use in radio-frequency channels are not well-suited for high-data-rate ACOMMS applications [2]. However, substantial progress has been made in ACOMMS since the early 1990's, leading to a set of algorithms to better deconvolve the long channel response with sparse filters [4-6], compensate for Doppler effects using iterative techniques $[7,8]$, and track time-varying channels with adaptive algorithms [2,9-10]. However, even with sophisticated processing routines, in practice it is often difficult to coherently recombine all paths. The uncombined paths will contribute incoherent interference that will limit the receiver [11]. Filtering out these residual paths can increase channel capacity by lowering the signal-to-reverberation noise floor closer to the signal-to-ambient noise limit.

\footnotetext{
${ }^{a}$ Note that bandwidths and center frequencies are typically decreased with range due to absorption (see Figure 1).
} 
One of the most effective ways to filter unwanted paths is in the spatial domain with a large aperture receiver pointed toward the source [12]. In fact, many existing large arrays could be used as ACOMMS receivers. Often, these receivers have hundreds or thousands of channels, vastly increasing the complexity of the full space-time optimization problem.

In this paper, we present a sub-optimal space-time processing technique using delay-weight-sum beamforming in conjunction with monopulse processing to suppress incoherent paths. This method substantially reduces equalizer complexity. In Section II, we present a general ACOMMS channel model with multipath and Doppler effects. In Section III, we present the wideband monopulse ACOMMS receiver and associated signal processing methods. In Section IV, we present a physical simulation of a simplified shallow water channel. In Section V, we present shallow water experimental data which supports our observations in Section IV. Finally, we state our conclusions in Section VI.

\section{ACOMMS CHANNEL MODEL}

The pressure waveform at the receiver can be modeled as a superposition of propagation paths (eigen-rays) that are delayed and affected by Doppler, resulting in a signal $y(t)$ that is doubly spread and scaled in both time and frequency,

$$
y(t)=\sum_{i=1}^{N_{p}} a_{i} x\left(\frac{1}{d_{i}} t+\Delta_{i}\right)+n(t) .
$$

Here, $N_{p}$ is the total number of paths, $n(t)$ is the noise amplitude at time $t$, and the remaining terms are characteristics of the $i^{\text {th }}$ path $-a_{i}$ is the amplitude, $d_{i}$ is the Doppler factor, and $\Delta_{i}$ is the delay. Note that due to the wideband nature of most underwater acoustic channels, Doppler effects are not best modeled as a frequency shift (i.e. phase modulation). Time/frequency dilation effects need to be considered [13].

The $i^{\text {th }}$ Doppler factor $d_{i}$ can be approximated by projecting the relative velocity vector (between the receiver and the source) $\vec{v}=\vec{v}_{r}-\vec{v}_{s}$ onto the $i^{\text {th }}$ position vector, $\vec{p}_{i}$, pointing from the receiver to the $i^{\text {th }}$ image of the source,

$$
d_{i}=1+\frac{\vec{v} \cdot \vec{p}_{i}}{\left|\vec{p}_{i}\right| c}
$$

Using the $i^{\text {th }}$ Doppler factor, the length of the $i^{\text {th }}$ path vector, and the speed of sound in water, $c$ (assumed constant), the $i^{\text {th }}$ delay can be computed,

$$
\Delta_{i}=\frac{\left|\vec{p}_{i}\right|}{c d_{i}}
$$

The $i^{\text {th }}$ path amplitude is reduced by reflection, geometrical spreading and absorption. As shown in (4), the $i^{\text {th }}$ path amplitude is the product of all $N_{r}$ reflection coefficients, $\Gamma_{r}$, the wavelength dependent geometrical spreading expression

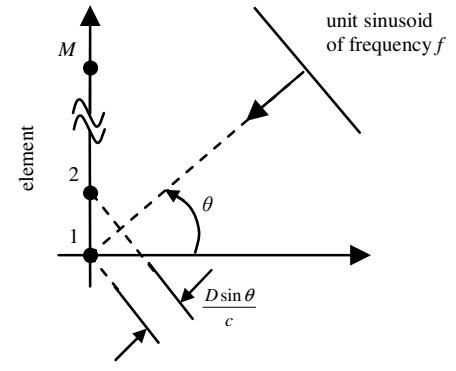

Figure 2. An $M$-element uniformly-spaced line array of inter-element spacing $D$ is illuminated by a plane wave arriving from a source at angle $\theta$ from broadside. Elements can be progressively delayed by $D \sin \theta / c$ and summed to maximize response at angle $\theta$.

with geometry factor $k$ (where $k=1$ for spherical), and the absorption loss (assuming constant absorption coefficient $\alpha$ over frequency and time),

$$
a_{i}=\left(\prod_{r=1}^{N_{r}} \Gamma_{r}\right)\left(\frac{\lambda}{4 \pi\left|p_{i}\right|^{k}}\right) e^{-\alpha\left|p_{i}\right|} .
$$

Note that there are more complicated effects in physical channels that have been neglected-e.g. time-varying path parameters, wavelength-dependent absorption and sound speed, and Doppler effects that change over time due to platform acceleration [14].

\section{WIDEBAND MONOPULSE ACOMMS RECEIVER}

Monopulse processing is a technique used to improve the angular precision of tracking systems to a value finer than the beamwidth of the seeing aperture. This has the advantage of allowing for more compact tracking systems since the beamwidth of an aperture is physically constrained by its cross-section in wavelengths (i.e. beamwidth within a given plane is $\sim 1.22 \lambda / B$, where $B$ is the baseline of the aperture in that plane). The wideband monopulse method, also known as the space-time filtered gradient method, is an extension of classical sum-and-difference monopulse for use in wideband systems [15-17]. A comprehensive review of direction finding methods and monopulse processing is given in [18].

A discrete array of sensors can be used to sample an acoustic field across a given aperture. It can be steered to give an output that is most sensitive for plane waves arriving from a particular direction by the following steps: (i) delay each element output such that plane waves from that direction arrive simultaneously and (ii) add those delayed outputs. The resulting angular response pattern can be tailored by applying weights prior to summing. For this reason, the resulting "beam output," $s(t)$, is said to be formed by a delay-weightsum process. This process is most approachable when considering an $M$-element uniformly-spaced line array of inter-element spacing $D$ such as the one shown in Figure 2. Here, a beam can be steered toward a unit sinusoid of frequency $f$ at angle $\theta_{s}$ by summing all elements outputs $x_{i}(t)$ with a progressive phase delay and corresponding weight $w_{i}$ 
(chosen for desired beampatterns-e.g. edges tapered for reduced sidelobes [19]):

$$
y(t)=\sum_{i=1}^{M} w_{i} x_{i}(t) e^{j 2 \pi f(i-1) \frac{D \sin \theta_{s}}{c}} .
$$

On the other hand, for wideband signals, phase shifts proportional to frequency must be applied to achieve the required delay. This leads to the result:

$$
y(t)=\int_{-\infty}^{\infty}\left(\sum_{i=1}^{M} w_{i} X_{i}(f) e^{-j 2 \pi f(i-1) \frac{D \sin \theta}{c}}\right) e^{j 2 \pi f t} d f .
$$

Here, $X_{i}(f)$ denotes the continuous Fourier transform of the element output $x_{i}(t)$. A discrete Fourier transform is commonly used in practice, as element outputs are typically digitally sampled after being pre-amplified and conditioned. Discrete-time processing methods are then used. Though many factors must be considered for practical array design (e.g. minimum spatial sampling to avoid aliased response patterns, or grating lobes, element response, variability and coupling, etc.), the delay-weight-sum beamforming process can be applied in this manner.

A wide array needs many azimuthally-narrow, steered beams to cover the field of view (see Figure 3 ). To limit the complexity of vertical beamforming, wideband monopulse processing generates a pair of beam outputs, $s_{0}(t)$ and $s_{1}(t)$, as discussed in [15-17], with the following linear relationship:

$$
s_{1}(t) \approx\left(\sin \theta-\sin \theta_{s}\right) \cdot s_{0}(t)
$$

Here, $\theta$ is the elevation angle of the source and $\theta_{s}$ is the vertical beam steering angle. Two uses of (7) are (i) direction finding and (ii) null steering. These beam outputs $s_{0}(t)$ and $s_{1}(t)$ can be generated in five steps: (i) assume the array is a uniformly-spaced vertical stack of $M$ identical sub-arrays forming identical azimuthal beams, (ii) form $w_{b}$, a vector of $M-1$ shading coefficients selected for desired vertical beam patterns (e.g. a Hanning window), (iii) form two $M$-element shading vectors $w_{0}=[1,1] \otimes w_{b}$ and $w_{1}=[1,-1] \otimes w_{b}$, where $\otimes$ denotes discrete convolution, (iv) form two beam outputs by applying $w_{0}$ and $w_{1}$ to the $M$ sub-arrays using classical delayweight-sum beamforming for vertical steering angle $\theta_{s}$, and (v) apply filters $h_{0}(t)$ and $h_{1}(t)$ to those beam outputs, where $h_{0}(t)$ and $h_{1}(t)$ have the following relationship:

$$
h_{0}(t)=\left(\frac{D}{2 c}\right) \frac{d}{d t} h_{1}(t)
$$

Here, $D$ is the spacing between elements and $c$ is the wave propagation speed in the medium of interest. These steps give the frequency domain relationship (this justifies (7)):

$$
\frac{S_{1}(f)}{S_{0}(f)}=\frac{j \tan \left[\pi f(D / c)\left(\sin \theta-\sin \theta_{s}\right)\right]}{j \pi f(D / c)} \approx \sin \theta-\sin \theta_{s} .
$$
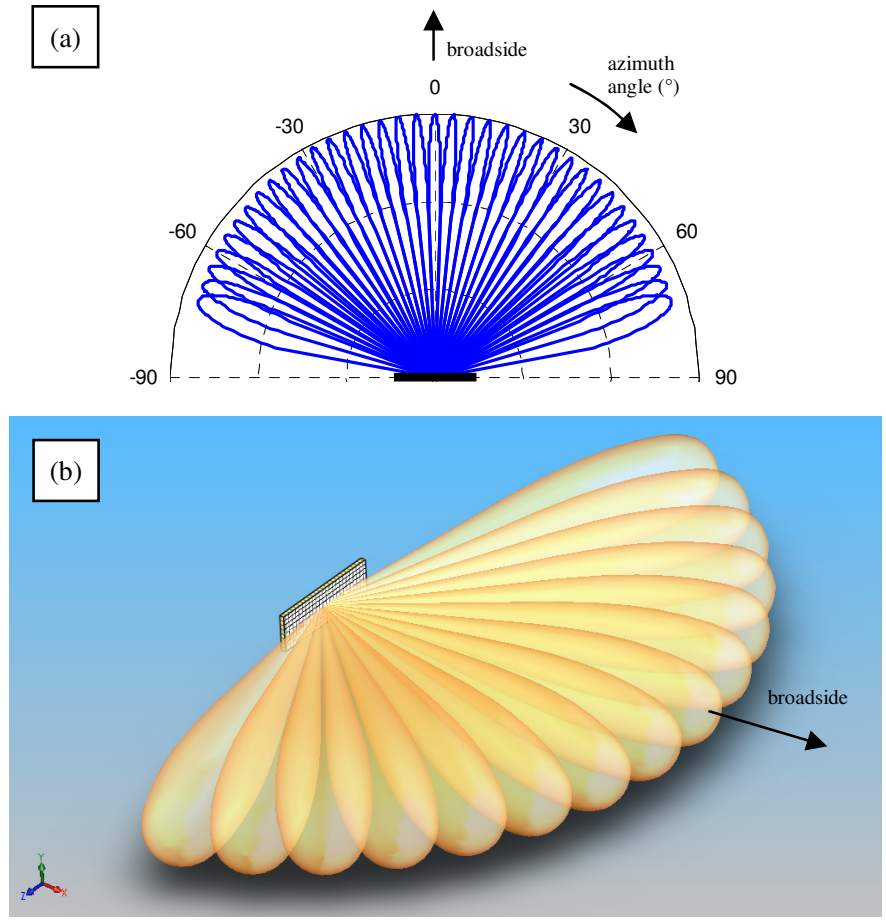

Figure 3. (3a) An overhead view of a fan of narrow azimuthal beams produced by delay-weight-sum beamforming a wide array (array shown in heavy black, $20 \mathrm{~dB} / \mathrm{div}$ ). (3b) A 3-d rendering of a fan of azimuth beams.

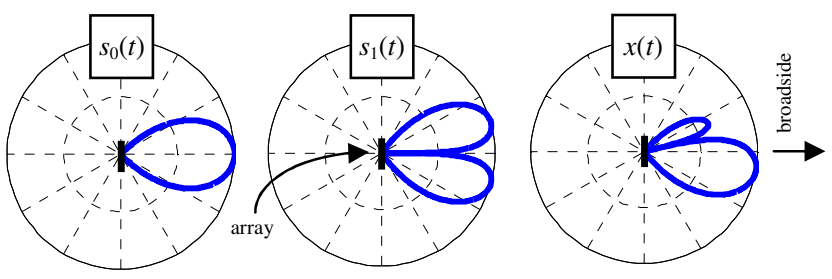

Figure 4. Broadband theoretical beam patterns showing angular sensitivity of a six-element vertical line array (heavy black) with $\lambda / 2$ spacing and quality factor $Q=4$. From left to right $-s_{0}(t), s_{1}(t)$, and their linear combination, $\mathrm{x}(t)$ for $\theta_{n}=10^{\circ}$ from broadside - are plotted in log scale $(20 \mathrm{~dB} / \mathrm{div})$.

A beam with a broadband (vertical) null at elevation angle $\theta_{n}$ can be formed through a linear combination of $s_{1}(t)$ and $s_{0}(t): x(t)=s_{1}(t)-\left(\sin \theta_{n}-\sin \theta_{s}\right) \approx \alpha s_{0}(t)$, where $\alpha=\sin \theta-$ $\sin \theta_{n}$. Therefore, $s_{1}(t)$ and $s_{0}(t)$ can be fed as separate inputs to a linear, multichannel equalizer. The equalizer is then allowed to form this same linear combination (if it wants to), thereby nulling broadband interference at a dynamically varying $\theta_{n}$. The theoretical wideband vertical beampatterns of a six-element vertical line array with $\lambda / 2$ spacing and quality factor $Q=4$ are shown in Figure 4. In this figure, $s_{0}(t)$ and $s_{1}(t)$ are linearly combined to steer a wideband null to $\theta_{n}=10^{\circ}$ (i.e. $x(t) \approx s_{1}(t)-0.17 s_{0}(t)$ ). This process can be extended to form additional monopulse pairs with the same relationship. For example, one can form three beam outputs, $s_{0}(t), s_{1}(t)$, and $s_{2}(t)$ such that this linear relationship,

$$
s_{2}(t) \approx\left(\sin \theta-\sin \theta_{s}\right) \cdot s_{1}(t)
$$

applies in addition to (7). 
Now, we present a complete ACOMMS system which uses wideband monopulse processing. Portions of the receiver structure are based on works presented in [7-9,11-12]. The structure of the single-carrier communication waveforms used for the receiver is shown in Figure 5. The waveform consists of a linear frequency modulation (LFM) sweep over the transmit bandwidth used for detection and synchronization followed by a silent period to let reverberation decay. LFMs are useful because they retain strong auto-correlation properties under Doppler distortion [20]. The data payload consists of 4096 pseudo-random symbols encoded using various modulation schemes, a portion of which are used for Doppler detection and equalizer training. The waveforms encoded with 16QAM modulation are the focus of this paper.

A system diagram summarizing the wideband monopulse ACOMMS receiver processing is shown in Figure 6. As shown in the figure, the pressure waveform incident upon the receive array is converted into a voltage signal at each element, conditioned, then digitized. The digital array samples are then processed using a delay-weight-sum beamformer to form a fan of narrow azimuthal beams. Transmitted signals are detected and time-registered by matched-filtering the samples with a replica of the transmitted LFM sweep. The array is then steered toward the most correlated azimuth angle and vertically processed to yield monopulse channels.

The bulk Doppler is then estimated by cross-correlating the $s_{0}(t)$ waveform with a bank of Doppler-distorted replicas of the training symbols. The highest output is the bulk Doppler estimate and the samples of the $s_{0}(t)$ and $s_{1}(t)$ waveforms are compensated by dilating the time axis using an interpolating filter. The Doppler-compensated and synchronized beam outputs are then filtered and combined using a multichannel, fractionally-spaced decision-feedback equalizer with 11 uniformly-spaced forward taps per channel and 5 uniformlyspaced feedback taps. The equalizer tap coefficients are initialized using a direct least-squares (LS) estimate over the training sequence with no prior knowledge of the channel. Its taps are then updated using a decision-directed least-meansquares (LMS) algorithm over the data. Equalizer adaptation rates for results reported in this paper are 0 and 0.01 . More on equalization for underwater channels can be found in [21].

\section{SiMULATION}

To evaluate the performance of the monopulse ACOMMS receiver, a simulation of a simplified physical channel was constructed based on the model discussed in Section II. For a narrow azimuthal beam, the shallow ACOMMS channel can be simplified into a 2-D vertical plane of water which contains the receiver and the source. This plane is intersected by two infinite horizontal boundaries-i.e. the surface and the bottom. The simplified physics allows us to obtain quantities for the path parameters discussed in Section II. The $r^{\text {th }}$ reflection coefficient can be calculated using the Rayleigh formula [22]:

$$
\Gamma_{r}=\frac{z_{1,3} / z_{2}-\sqrt{1-[n-1] \tan ^{2} \phi_{i}}}{z_{1,3} / z_{2}+\sqrt{1-[n-1] \tan ^{2} \phi_{i}}} .
$$

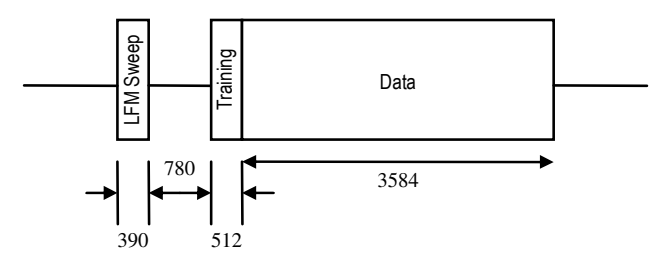

Figure 5. The waveform structure with the length of each section denoted in symbol periods. The waveform begins with a Doppler-tolerant linear frequency modulation (LFM) sweep followed by a period of silence and 4096 pseudo-random data symbols encoded with 16QAM. A fraction of the data symbols (training) are known to the receiver.

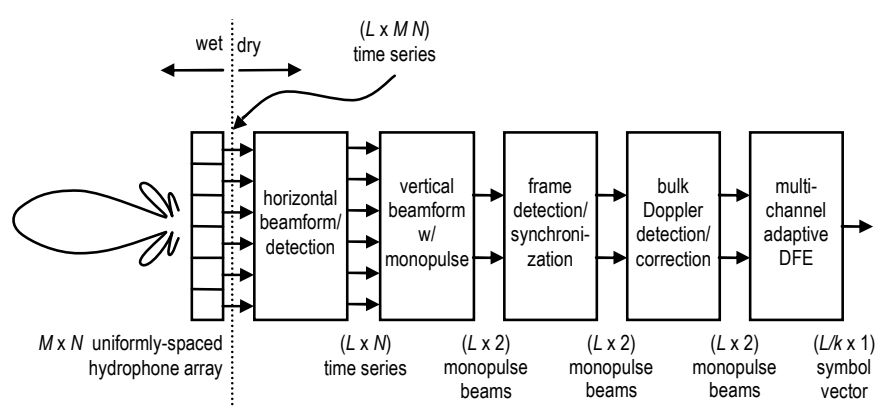

Figure 6. System block diagram of the wideband monopulse ACOMMS receiver. Analog voltages from a $M \times N$ uniformly spaced hydrophone array are conditioned and digitized then horizontally beamformed over a fan of azimuth angles. The array is then steered toward the most coherent azimuth direction and vertically processed to yield the beam pair $s_{0}(t)$ and $s_{1}(t)$. Channel delay and Doppler distortion are compensated, and both channels are recombined using a multichannel adaptive DFE.

TABLE II

Physical ChanNel Simulation SPECIFICATIONS

\begin{tabular}{|c|c|}
\hline Parameter & Value(s) used \\
\hline ranges $(\mathrm{m})$ & $25: 25: 700$ \\
\hline transmitter depth $(\mathrm{m})$ & 10 \\
\hline receiver depth $(\mathrm{m})$ & 5 \\
\hline transmitter closing speed $(\mathbf{m} / \mathbf{s})^{\mathrm{b}}$ & $\operatorname{sim} 1=0 / \operatorname{sim} 2=5$ \\
\hline sound speed in medium $1, c_{1}\left(\mathrm{~m} \cdot \mathrm{s}^{-1}\right)$ & 343 \\
\hline acoustic impedance of medium $1, z_{1}\left(\mathrm{~N} \cdot \mathrm{s} \cdot \mathrm{m}^{-3}\right)$ & 415 \\
\hline sound speed in medium $2, c_{2}\left(\mathrm{~m} \cdot \mathrm{s}^{-1}\right)$ & 1500 \\
\hline acoustic impedance of medium $2, z_{2}\left(\mathrm{~N} \cdot \mathrm{s} \cdot \mathrm{m}^{-3}\right)$ & $1,480,000$ \\
\hline sound speed in medium $3, c_{3}\left(\mathrm{~m} \cdot \mathrm{s}^{-1}\right)$ & 1500 \\
\hline acoustic impedance of medium $3, z_{3}\left(\mathrm{~N} \cdot \mathrm{s} \cdot \mathrm{m}^{-3}\right)$ & $2,324,000[23]$ \\
\hline maximum number of reflections, $N_{r-\max }$ & 1 \\
\hline ambient noise power, $N_{0}$ (dB re: direct path) & -20 at $700 \mathrm{~m}$ \\
\hline DFE adaptation rate $\mu^{\mathrm{b}}$ & $\begin{aligned} \operatorname{sim} 1 & =0.00 / \\
\operatorname{sim} 2 & =0.01\end{aligned}$ \\
\hline number of $(1 / 2)$-spaced forward taps per channel & 11 \\
\hline number of (1)-spaced decision feedback taps & 5 \\
\hline
\end{tabular}

Here, $z_{1,3}$ is the acoustic impedance of the surface or bottom media depending on which boundary the ray encounters (e.g. air or wet sand), $z_{2}$ is the acoustic impedance of the water, $\phi_{i}$ is the incident angle with respect to the normal to the boundary, and $n=\left(c_{1,3} / c_{2}\right)^{2}$, where $c_{1,3}$ is the sound propagation speed of the surface or bottom media and $c_{2}$ is the sound speed in water. Boundary parameters for the simulation are shown in Table 2. For this case, $\Gamma_{r}$ is always real. Typical reflection coefficients using these values for the surface and bottom are about -1 and 0.2 at normal incidence, respectively.

${ }^{b}$ Only the parameters shown in bold were changed between simulations. Scenario 1 is denoted by "sim1" and scenario 2 is denoted by "sim2." 
The math can further be simplified by neglecting refractive effects within the propagation media by assuming it is isotropic (i.e. constant sound speed). This allows boundary incident angles to remain constant. Using this generalization, the point source transmitter is imaged by reflecting its position with respect to the boundaries $N_{r}$ times for a path with $N_{r}$ reflections, allowing $\vec{p}_{i}$ to be readily calculated. The maximum number of reflections, $N_{r-\max }$, is a simulation input. The $i^{\text {th }}$ delay, $\Delta_{i}$, and Doppler factor, $d_{i}$, follow from $\vec{p}_{i}$ as described in Section II. The propagation paths under these conditions for a $75 \mathrm{~m}$ isotropic channel with $N_{r-\max }$ equal to 5 are shown in Figure 5.

Using this simulation, we model two challenging multipath scenarios:

1. Stationary transmitter broadcasting to a 6-element line receiver of the type discussed in Section III. Range is varied from $25 \mathrm{~m}$ to $700 \mathrm{~m}$ in $25 \mathrm{~m}$ steps. This simulation demonstrates that, using monopulse processing, the multichannel equalizer can control the linear combination of the monopulse pairs to suppress incoherent multipath arriving outside of its 'visible' time-span.

2. Same as simulation 1 but with a roving transmitter travelling at modest speeds (i.e. $5 \mathrm{~m} / \mathrm{s}$ ). This simulation demonstrates that monopulse processing can be used in combination with the multichannel equalizer to suppress incoherent Doppler-spread paths arriving within the 'visible' time-span of the equalizer.

To evaluate the performance of the receiver, we compute the output signal to noise ratio $(O S N R)$, in $\mathrm{dB}$, at the output of the equalizer:

$$
\operatorname{OSNR}[d B]=10 \log _{10}\left(\frac{\overline{(\hat{x}-\bar{x})(\hat{x}-\overline{\hat{x}})^{*}}}{\overline{(\hat{y}-\hat{x}-\overline{\hat{y}-\hat{x}})(\hat{y}-\hat{x}-\overline{\hat{y}-\hat{x}})^{*}}}\right) .
$$

Here, an overbar denotes the sample mean, $\hat{x}$ is the transmitted symbol vector normalized to unit energy, and $\hat{y}$ is the symbol vector output by the multichannel equalizer. OSNR is decreased by both the additive noise of the channel, inter-symbol interference (ISI) caused by incoherent multipath, and uncompensated Doppler effects.

\section{A. Scenario 1 - Stationary Transmitter and Receiver}

For this simulation, the transmitter is at $5 \mathrm{~m}$ depth and the receiver line array is centered at $10 \mathrm{~m}$ depth. Both platforms are stationary, but the distance between the platforms is varied from 25 to $700 \mathrm{~m}$ in $25 \mathrm{~m}$ steps. The maximum number of reflections is set to $1\left(N_{r-\max }=1\right)$, allowing for one surface and one bottom bounce. The adaptation factor for this test $(\mu)$ was set to 0.00 (i.e. no adaptation). The values for the remaining parameters are shown in Table 2. Four receiver configurations were used for the test: (i) 1-ch equalizer processing $s_{0}(t)$, (ii) 2-ch equalizer processing $s_{0}(t)$ and $s_{1}(t)$, (iii) 3-ch equalizer processing $s_{0}(t), s_{1}(t)$, and $s_{2}(t)$, and (iv) a 6-ch equalizer operating on the element outputs. The equalizer OSNR was recorded for each run. The results are shown in Figure 8(a/b).

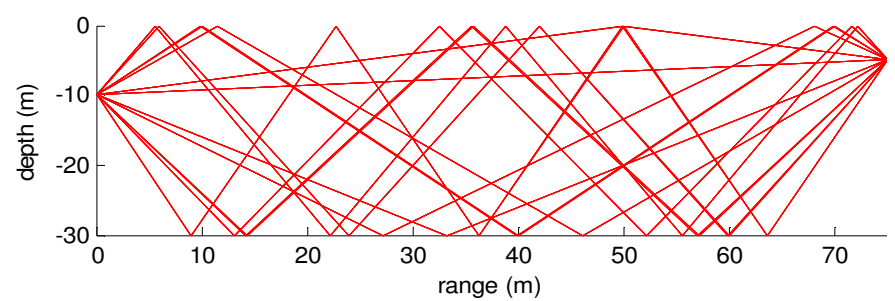

Figure 7. Simulation ray trace from transmitter (left) to receive array (right). In this figure, the maximum number of reflections, $N_{r-\max }$, equals 5.

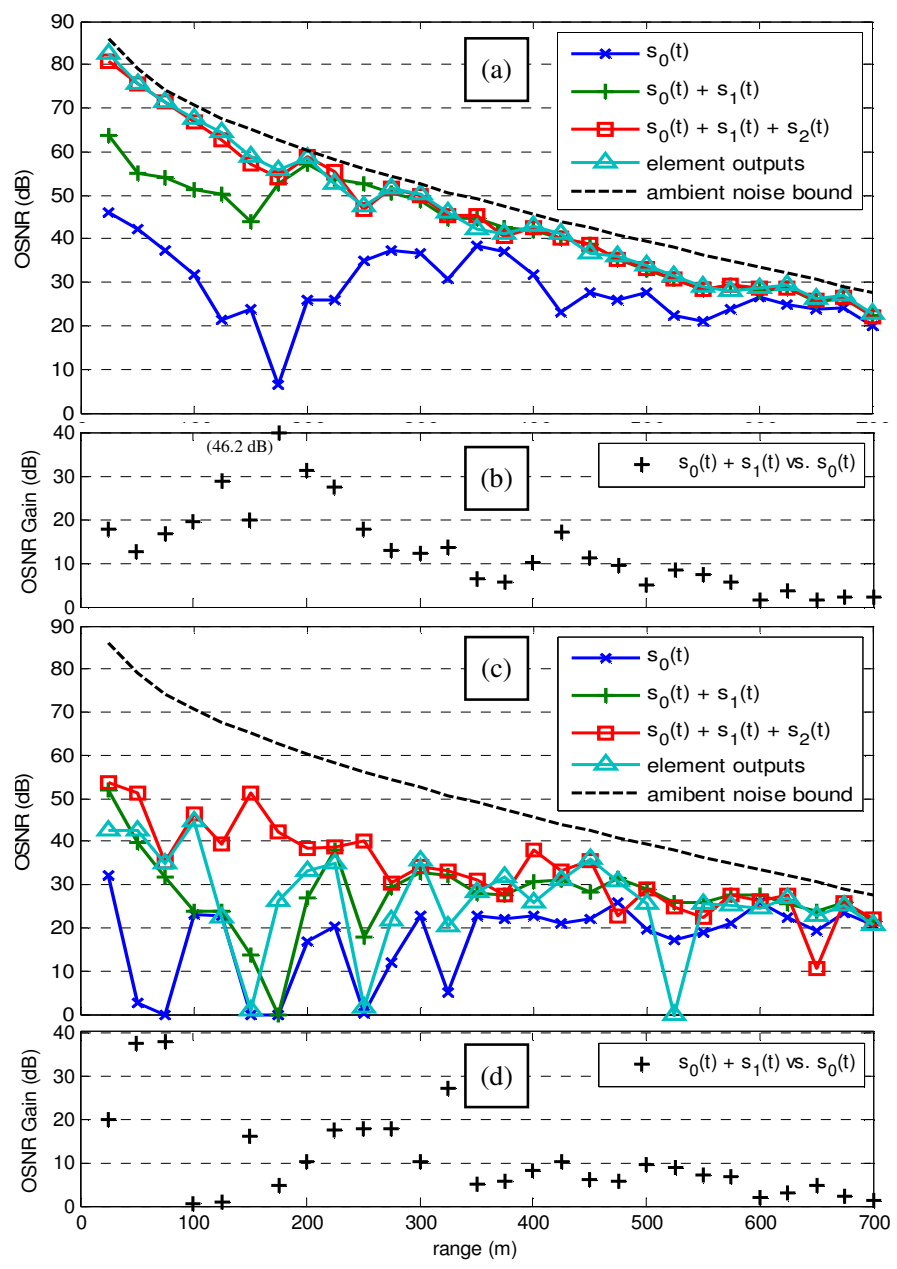

Figure 8. OSNR vs. range for communication scenario 1 and 2 in simulated channel. The signal to ambient noise bound $(27.8 \mathrm{~dB}$ at $700 \mathrm{~m})$ is denoted by the heavy dashed line. In simulation $1(8 \mathrm{a} / \mathrm{b})$, monopulse channels allow for nulls to be steered at interfering paths outside the 'visible' time-span of the equalizer. The 3-output monopulse ( 2 free nulls) performs nearly as well (and better at some ranges) as the 6 -ch equalizer at $\sim 1 / 8^{\text {th }}$ the computation cost. In scenario $2(8 \mathrm{c} / \mathrm{d})$, Doppler spread is severe, reducing the coherency of paths even when they lie within the 'visible' time-span of the equalizer. Since the equalizer cannot track and coherently recombine Doppler-spread paths, it is most beneficial to suppress them with spatial filtering, increasing OSNR.

As seen in the figure, equalizer OSNR for all four configurations varies with range due to the different delays and strengths of multipath echoes of each geometric environment. Using the $s_{0}(t)$ output ( $\mathrm{a} \sim 20^{\circ}$ wide beam aimed at $\theta_{s}=0^{\circ}$ ), the surface and bottom echoes strongly interfere with the direct path at certain ranges, particularly at 100-200 $\mathrm{m}, 325 \mathrm{~m}$, and $425 \mathrm{~m}$. Using this configuration, the equalizer 
is only able to suppress ISI when the delay lies within the time-span of the forward or feedback filters. The longest delay was on the order of a few hundred symbol periods observed at the closest range and the smallest delay was on the order of a few symbol periods at the furthest range.

Using the wideband null made available from preprocessing the array, the second configuration is able to suppress arrivals from a given direction, resulting in substantially better performance than using $s_{0}(t)$ alone. At $25-150 \mathrm{~m}$, the equalizer steers the null toward the surface. Beyond $150 \mathrm{~m}$, the surface echo arrives within the visible span of the equalizer and is able to be recombined by the filter taps. The primary interferer beyond this range is the bottom echo.

If the array is processed more extensively to yield $s_{2}(t)$ of (10), another free null is added. This allows the equalizer to null both the surface and bottom echoes at range $<150 \mathrm{~m}$, reducing ISI and allowing the receiver to approach the ambient noise limit. Allowing the equalizer to operate directly on the element outputs results in a marginal increase in signal quality over the third configuration $\left(s_{0}(t)+s_{1}(t)+s_{2}(t)\right)$ for most ranges (though not for every) at a large increase in complexity-e.g. doubling the size of the equalizer increases the operations required to invert the coefficient initialization matrix by $8\left(O\left(n^{3}\right)\right)$ and slightly increases the operations in the least-mean-squares adaptive stage. Computational savings would be even greater with a recursive-least-squares (RLS) algorithm since complexity is near $O\left(n^{3}\right)$ for the length of the packet [24].

One of the reasons why the element-level equalizer often outperforms the monopulse processed outputs is that it is able to optimize the filter coefficients to maximize the OSNR, allowing it to have full control over the spatial and temporal filtering. The reason why the element-level equalizer did not outperform monopulse processing at $250 \mathrm{~m}, 375 \mathrm{~m}$, and a few other ranges is that the equalizer's length may not have been sufficient to steer a deep wideband null at the interfering sources. For reference, for a 6-element line array, the time domain filtering discussed in Section III can be approximated by a 21-tap finite impulse response (FIR) filter that operates on each element's baseband signal (at twice the symbol rate).

\section{Scenario 2 - Roving Transmitter and Stationary Receiver}

This simulation follows the same configuration as Scenario 1 except that the transmitter is now in motion at a closing speed of $5 \mathrm{~m} / \mathrm{s}$ and the equalizer performs decision-directed adaptation over the course of the packet (with adaptation factor $\mu=0.01$ ). As in scenario 1 , the increased number of monopulse channels allows for more rejection of incoherent multipath. In this case, multipath is incoherent due to Doppler differences with the direct path while using only a single compensation factor. The difference between the OSNR and the ambient noise bound decreases with range for most configurations since the difference between the Doppler distortion in the direct path and the multipath is reduced. This is most apparent in the similarity between the furthest ranges in Figure 8(c/d) and Figure 8(a/b), which are nearly identical.

Another observation is that as range increases, the delay between the direct path and the surface and bottom

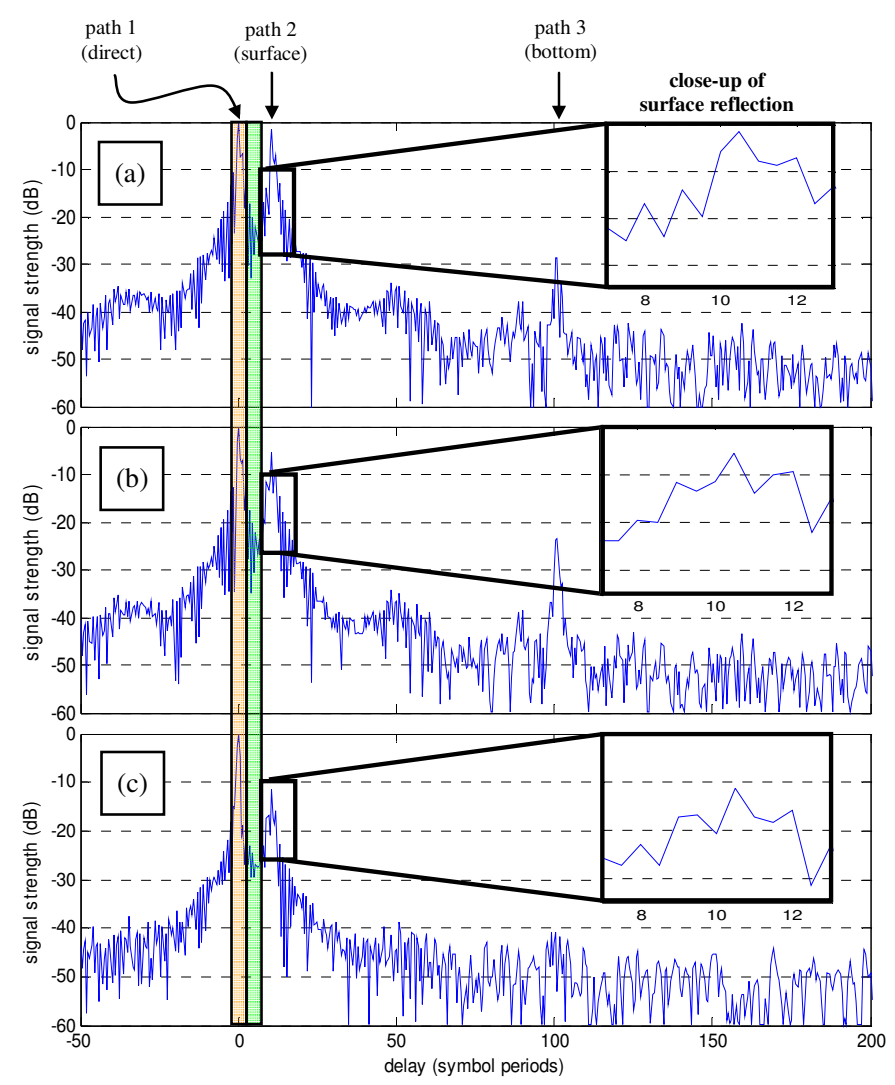

Figure 9. Channel impulse response of $s_{0}(t)(9 \mathrm{a})$, the linear combination of $s_{0}(t)$ and $s_{1}(t)$ using forward equalizer coefficients $(9 \mathrm{~b})$, and the linear combination of $s_{0}(t), s_{1}(t)$, and $s_{2}(t)$ combined in the same manner $(9 \mathrm{c})$. The close-ups in (9a) and (9c) show the $\sim 10 \mathrm{~dB}$ interference reduction outside of the equalizer's reach afforded by monopulse processing. The colored bars show the 'visible' time-span of the forward and decision-feedback taps.

reflection(s) is reduced, though their strength increases as incident angle approaches grazing. A visualization of incoherent multipath suppression is given in Figure 9. Here, a matched filtering operation with the transmitted LFM sweep reveals two paths which arrive outside of the time-span of the forward and decision-feedback equalizer taps. Using the linear combination of $s_{0}(t)$ and $s_{1}(t)$, the surface reflection is suppressed as shown in Figure 9(b). Further, adding the $s_{2}(t)$ beam (as shown in Figure 9(c)) allows for a linear combination to suppress both interferers, reducing ISI and improving OSNR.

\section{EXPERIMENTAL DATA}

Real data for this study was collected in the fall of 2009 at the Lake Travis Test Station (LTTS), a test facility located on Lake Travis, a shallow reservoir located about 20 miles northwest of downtown Austin, TX. For the experiment, an array receiver was mounted on a stationary platform at a depth of 16 feet. The array receiver was wide horizontally and short vertically with horizontal and vertical half-power beamwidths of approximately $3^{\circ}$ and $25^{\circ}$, respectively. A transmitting omnidirectional hydrophone was hung from the deck of a small research vessel that proceeded to various locations around the lake, sampling ranges from 25 to $700 \mathrm{~m}$. At each 
position, the hydrophone was lowered into the water at a rate of 2-3 meters per minute to a maximum depth of $10 \mathrm{~m}$. Transmissions took place the whole time the transmitter was submerged; thus, various depths between 0 and $10 \mathrm{~m}$ were sampled. The temperature and sound velocity profile of the lake were relatively constant during the time of the test as shown in Figure 10.

At each range/depth, several packet types were continuously transmitted in series. This study focuses on the 16QAM packet type. Element-level data was recorded from the array and later post-processed in MATLAB. Following the same processing routine outlined in Section III, the array was steered towards the azimuth of the transmitter and vertical monopulse beams were generated. The array was steered to $0^{\circ}$ elevation $\left(\theta_{s}=0^{\circ}\right)$. The beam pairs were then filtered and combined using the equalizer structure discussed in Section IV.

Figure 11 shows the OSNR gain of the monopulse receiver $\left(s_{0}(t)+s_{1}(t)\right)$ vs. $s_{0}(t)$. This was done to compare the gain of adding the $s_{1}(t)$ beam. Each black plus corresponds to a decoded packet. The most apparent gains are seen for the 26 packets recorded at the $75 \mathrm{~m}$ range (Experiment \#31-56). Gains of a few $\mathrm{dB}$ are seen throughout other ranges with some variability. Recall that depths were gradually varied from 0 $10 \mathrm{~m}$ then from $10-0 \mathrm{~m}$ at each range. Gains below zero $\mathrm{dB}$ are attributed to doubling the number of equalizer taps while keeping the adaptation factor constant in the presence of uncompensated Doppler. It is apparent that observed gains are highly dependant on range and depth. This is in agreement with the simulations of Section IV. Gains in other communication scenarios will depend on multipath environment and platform motion.

Figure 12 shows the mean OSNR gain of an 8-element vertical line array vs. a single-element receiver from a different experiment. Several different processing methods were compared. This data was collected under similar conditions as the 6-element data. Only packets with BER > 0.05 for all five methods were included (including singleelement). It is apparent in these mixed-condition experiments that the monopulse ACOMMS receiver outperforms straight beamforming yet is less complex and more agile than a receiver processing raw element outputs. Several important caveats of this technique should be montioned. First, when combining monopulse pairs, the overall beamwidth is widened. Though monopulse helps greatly in attenuating localized interferers, in the presence of uniformly distributed noise, it may perform worse due the decrease in beam directivity. Also, element variability (i.e. phase error, non-ideal placement, etc.) affects the depth of the null.

\section{CONCLUSION}

In this study, we have introduced the challenges of the underwater acoustic communication channel and discussed the mathematical complexities involved in processing space-time communication signals for a large receiver array. We have presented a model of the underwater acoustic communication (ACOMMS) channel which incorporates time-delayed and Doppler-spread multipath. We have discussed wideband

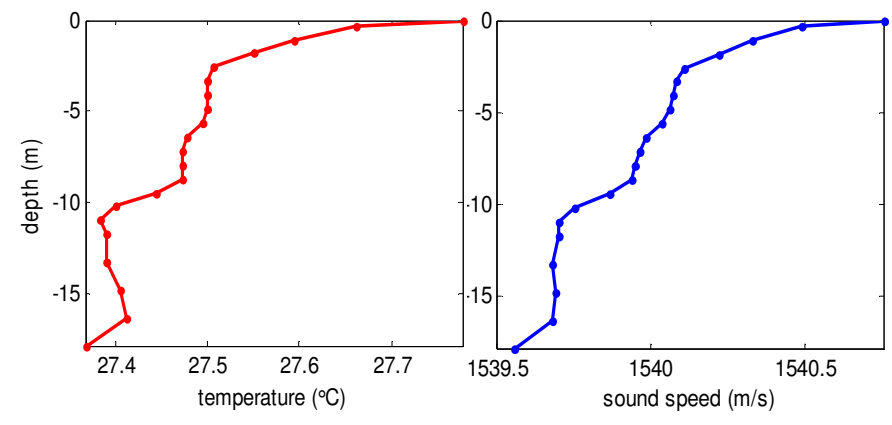

Figure 10. Temperature and sound velocity profiles for Fall 2009 lake test. Sound velocity calculated using formula presented in [25]. Acoustic waves propagating through water under these conditions will experience a mild downward refraction.

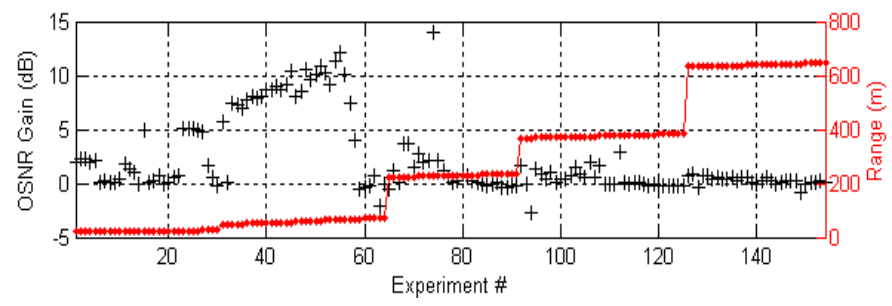

Figure 11. OSNR gain vs. range for monopulse ACOMMS receiver in a shallow water lake. Gain is given with respect to the OSNR of the $s_{0}(t)$ beam.

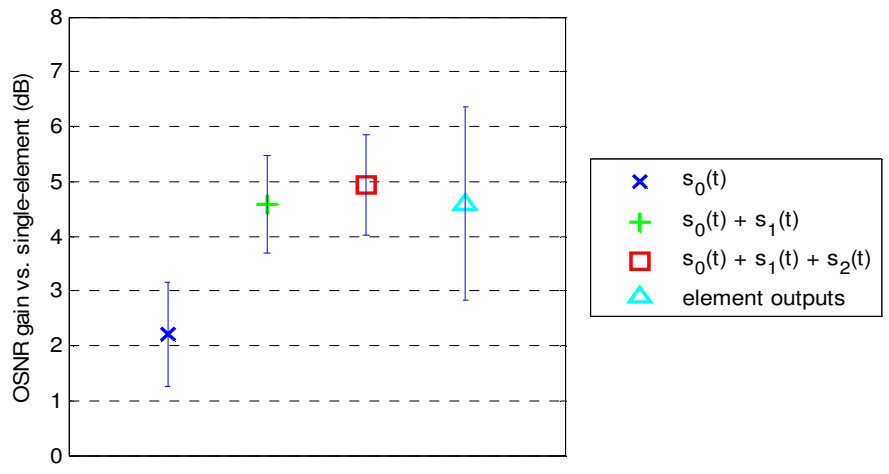

Figure 12. Mean OSNR gain and $\pm \sigma$ (error bars) of an 8-element vertical line array over a single element receiver (96 packets at various rates, $Q=0.8-3.1$ ).

monopulse processing and presented a receiver which utilizes its pre-processed beam outputs to steer wideband nulls at interference sources (boundary reverberation) with a scalar weight. We observe through both a simple geometric channel simulation as well as experimental data that the wideband monopulse ACOMMS receiver outperforms straight delayweight-sum beamforming $\left(s_{0}(t)\right)$. Monopulse processing allows for a simpler equalizer to dynamically steer nulls at unwanted paths at a fraction of the complexity of the optimal space-time methods. However, the observed gains are highly dependant on multipath environment (i.e. range, communicator depths, and motion) and will vary depending on field conditions.

\section{ACKNOWLEDGMENT}

The authors would like to thank ARL:UT's Independent Research and Development Program for supporting this work. 


\section{REFERENCES}

[1] R. J. Urick, "Principles of Underwater Sound," $3^{\text {rd }}$ edition, McGraw-Hill, Inc., New York, 1983.

[2] D. B. Kilfoyle and A. B. Baggeroer, "The state of the art in underwater acoustic telemetry," IEEE J. of Oceanic Eng., vol. 25, pp. 4-27, 2000.

[3] R. E. Francois and G. R. Garrison, "Sound absorption based on ocean measurements -2 . boric acid contribution and equation for total absorption," J. Acoust. Soc. Am., vol. 72, no. 6, pp. 1879-1890, 1982.

[4] M. Kocic, D, Brady, and M. Stojanovic, "Sparse equalization for realtime digital underwater acoustic communications," OCEANS '95. MTS/IEEE. Challenges of Our Changing Global Environment. Conference Proceedings, vol. 3, pp. 1417-1422, 1995.

[5] A. Chopra and B. L. Evans, "Design of sparse filters for channel shortening", Proc. IEEE Int. Conf. on Acoustics, Speech, and Signal Proc., pp. 1518-1521, 2010.

[6] M. J. Lopez, A. C. Singer, S. L. Whitney, and G. S. Edelson, "A DFE coefficient placement algorithm for underwater digital acoustic communications," OCEANS '99 MTS/IEEE. Riding the Crest into the 21 st Century, vol. 2, pp. 996-1001 vol. 2, 1999.

[7] P. J. Beaujean and L. R. LeBlanc, "Adaptive array processing for highspeed acoustic communication in shallow water," IEEE J. of Oceanic Engineering, vol. 29, no. 3, pp. 807-823, 2004.

[8] L. Freitag, M. Grund, J. Catipovic, D. Nogle, B. Pazol, and J. Glynn, "Acoustic communication with small UUVs using a hull-mounted conformal array," OCEANS, 2001., pp. 2270-2275, 2001.

[9] M. Stojanovic, J. A. Catipovic, and J. G. Proakis, "Phase-coherent digital communications for underwater acoustic channels," IEEE J. of Oceanic Engineering, vol. 19, no. 1, pp. 100-111, 1994.

[10] S. Hwang and P. Schniter, "Efficient multicarrier communication for highly spread underwater acoustic channels," IEEE J. on Sel. Areas in Comm., vol. 26, no. 9, pp. 1674-1683, 2008.

[11] D. Rouseff, M. Badiey, and A. Song, "Effects of reflected and refracted signals on coherent underwater communication: Results from the Kauai experiment (KauaiEx 2003)," J. Acoust. Soc. Am., vol. 126, no. 5, pp. 2359-2366, 2009.
[12] T. C. Yang, "A study of spatial processing gain in underwater acoustic communications," IEEE J. of Oceanic Eng., vol. 32, no. 3, 2007.

[13] D. W. Ricker, "Echo Signal Processing," Kluwer Academic Publishers Group, 2003.

[14] K. A. Perrine, K. F. Nieman, K. H. Lent, T. L. Henderson, T. J. Brudner, B. L. Evans, "Doppler estimation and correction for shallow underwater acoustic communications," Proc. Asilomar Conf. on Signals, Systems, and Computers, 2010.

[15] T. L. Henderson, "Matched beam theory for unambiguous broadband direction finding," J. Acoust. Soc. Am., vol. 78, no. 2, pp. 563-574, 1985.

[16] T. L. Henderson, "Wide-band monopulse sonar: processing performance in the remote profiling application," IEEE J. of Oceanic Eng., vol. 12, no. 1 , pp. 182-197, 1987

[17] T. L. Henderson and S. G. Lacker, "Seafloor profiling by a wideband sonar: simulation, frequency-response optimization, and results from a brief sea test," IEEE J. of Oceanic Eng., vol. 14, no. 1, pp. 94-107, 1989.

[18] T. L. Henderson and T. J. Brudner, "A space-time filtered gradient method for detecting directions of echoes and transient sounds," $J$. Acoust. Soc. Am. 118, pp. 679-695, 2005.

[19] C. A. Balanis, "Antenna Theory: Analysis and Design, $3^{\text {rd }}$ Edition," Wiley-Interscience, 2005.

[20] C. C. Tsimenidis, B. S. Sharif, O. R. Hinton, and A. E. Adams, "Analysis and modelling of experimental doubly-spread shallow-water acoustic channels," Oceans 2005 - Europe, vol. 2, pp. 854- 858, 2005.

[21] K. F. Nieman, K. A. Perrine, K. H. Lent, T. L. Henderson, T. J. Brudner, B. L. Evans, "Multi-stage and sparse equalizer design for communication systems in reverberant underwater channels," Proc. IEEE Int. Workshop on Signal Processing Systems, 2010.

[22] J. W. S. Rayleigh, "The Theory of Sound," Dover Publications, 1945.

[23] D. T. Smith and W. N. Li, "Echo-sounding and sea-floor sediments," Marine Geology, vol. 4, no. 5, pp. 353-364, 1966.

[24] F. A. Blackmon, W. Canto, "Performance comparison of several contemporary equalizer structures applied to selected field test data," OCEANS 2000 MTS/IEEE Conference, vol. 2, pp. 809-816, 2000.

[25] H. Medwin, "Speed of sound in water: A simple equation for realistic parameters," J. Acoust. Soc. Am., vol. 58, no. 6, pp. 1318-1319, 1975. 